INNOVATIONS IN PRIMARY CARE

\title{
Screening for Depression in Pregnancy-There's an App for That!
}

\author{
Sarab Ricketts, $M D^{1}$ \\ Tram N. B. Nguyen ${ }^{2}$ \\ Varsha Narasimban, $M D^{3}$ \\ ${ }^{1}$ Montefiore Care Management Organization, Yonkers, New York \\ ${ }^{2}$ Albert Einstein College of Medicine, Bronx, New York \\ ${ }^{3}$ Montefiore Medical Center, Bronx, New York \\ Ann Fam Med 2019;17:275. https://doi.org/10.1370/afm.2388.
}

\section{THE INNOVATION}

Depression during pregnancy or postpartum causes significant adverse biological and behavioral outcomes for mothers, fetuses, children, and families. Depression screening of peripartum women is not routinely done in many practice settings. ${ }^{1-3}$ Recognizing the value of smartphone applications (apps) to provide health and self-management information, we developed and implemented an app which screens for depression, substance use, and social determinants of health (SDH) interposed between short articles about pregnancy, fetal development, and wellness. ${ }^{4}$ Our app contains a bidirectional chat feature to support engagement.

\section{WHO AND WHERE}

We offered the HIPAA-compliant smartphone application to pregnant women at 2 Montefiore Bronx obstetrician-gynecologist (OBGYN) clinics. Eligible women were those under 28 weeks gestation who owned an Android or iOS smartphone and were fluent in English or Spanish. The HealthyMoms team, which includes care managers and a telephonic mental health care team, led the project and worked closely with onsite obstetrics providers.

\section{HOW}

Eligible women were identified through daily $O B$ appointment rosters. A care manager contacted the patient in person at

Conflicts of interest: authors report none.

\section{Corresponding author}

Sarah Ricketts, MD

Montefiore Care Management Organization

200 Corporate Blvd South

Yonkers, NY 10701

srickett@montefiore.org the clinic or by telephone. Women who were interested and consented were entered into the dashboard, and we provided instruction on downloading and using the app. In addition to weekly informative articles, 8-item patient health questionnaires (PHQ- 8$)^{5}$ were sent in each trimester and twice postpartum. Substance use and SDH questions were sent to patients at enrollment and 6 months later.

Women who screened positive for depression or substance use were referred for telephonic evaluation by the team mental health clinicians who provided information to the patient about their symptoms and described treatment options. These options included no-cost telephonic psychotherapy, as well as referrals to outpatient care. Immediate evaluation by a psychiatrist was available. If a patient elected not to enter treatment, the clinician made monthly check-in calls, and PHQ-8s were sent through the app monthly.

If a patient was struggling with housing, food, legal, or violence concerns, referrals to the appropriate agency were made through the application through the chat feature. The care manager relayed any concerning information to the OB providers.

\section{LEARNING}

We have reached our target enrollment of 100 women, with $75 \%$ of women approached agreeing to enroll. Of the patients enrolled, $77 \%$ have been active in the app. Fifty-four percent have completed PHQ-8 depression screening, and 10\% of those screened positive with a score of $\geq 10$. The percent of women who screen positive for depression is concordant with prospective research studies. ${ }^{2}$ Forty-six percent have completed the (SDH/substance abuse) screening. Electronic screening also confirmed our earlier findings that administration by self-report leads to higher screen-positive rates. Our rate is substantially higher than onsite screening efforts at sites throughout New York City.

We initially thought that enrollment would be best facilitated by in-person enrollment, but were surprised to find that telephonic enrollment is nearly as effective as meeting patients in person. These preliminary results show that integrating an innovative smartphone app into OB practice can improve depression screening in a pregnant vulnerable population and thus improve early identification and treatment.

For references, funding support and acknowledgments, please see http://www.AnnFamMed.org/content/17/3/275/suppI/DC1/.

Key words: smartphone application; depression; pregnancy; mood disorders; pregnancy complications; technological innovations 